\title{
Development of an experimental activity for teaching cooperativity and allosterism Manta B. ${ }^{1}$, Calcagno M.L. ${ }^{2}$ y Alvarez B. ${ }^{1}$
}

1-Laboratorio de Enzimología, Facultad de Ciencias, Universidad de la República, Uruguay. 2-Departamento de Bioquímica, Facultad de Medicina, UNAM, México.

Although enzyme control and regulation is an important topic in most Biochemistry and Enzymology courses, laboratory activities that allow an experimental approach to cooperativity and allosterism are difficult to implement. The objective of this work was to develop a simple and inexpensive experimental activity to teach this topic in basic courses. We decided to use the enzyme glucosamine-6-phosphate deaminase (GNPD, E.C. 3.5.99.6) from Escherichia coli, that is both kinetically and structurally well-known. GNPD is an allosteric enzyme, activated by $N$ acetylglucosamine 6-phosphate, that catalyzes the conversion of glucosamine 6-phosphate into fructose 6-phosphate and ammonia. The enzyme is a typical allosteric K-system and can be well described by the Monod-WymanChangeux (MWC) model. GNPD was partially purified through anionic-exchange chromatography from a mutant E.coli strain which expresses constitutively high levels of the enzyme. In order to measure activity we used an end point method which consists in stopping the reaction at a certain time point with $\mathrm{HCl} 10 \mathrm{~N}$, and quantifying the fructose-6-phosphate formed with resorcinol (Selliwanoff reaction) through the formation of a red color that is measured spectrophotometrically. We developed a protocol that consisted in a 4-hour experiment in which the students measured the activity of the GNPD with increasing concentrations of the substrate, in the presence or absence of allosteric modulator. The students obtained a good quality data set that they analyzed based on the equations of Hill, MWC and Acerenza-Mirzaji (Biochim Biophys Acta, 1997, 1339:155-66). This protocol was used during the 2005 Enzymology course in Montevideo with very satisfactory results. 